\title{
Entretien avec Pierre Léon
}

\author{
Claude Tatilon \\ Université York
}

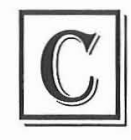

laude Tatilon. Cher Pierre, je soubaiterais que nous nous entretenions de ton petit dernier, Humour en coin. C'est un livre qui a eu un franc succès puisque ton éditeur en a fait un second tirage six mois après sa parution. Qu'est-ce qui t'a poussé à écrire ce gros volume? Et d'où vient le titre?

Pierre Léon. Oui, je pense que le livre a plu. Mais le premier tirage était modeste. Le deuxième a permis d'avoir une meilleure impression des illustrations et de corriger quelques coquilles!

Le titre? Ce sont des chroniques de faits d'actualité, demandées par L'Express de Toronto, et qui ont tourné à l'humour. Je ne me voyais pas raconter sans mettre un grain de sel pour amuser le chaland. Les rédacteurs successifs ont pris l'habitude de mettre cette chronique dans le coin du haut de la deuxième page. Ça tombait bien parce qu'il y a dans l'usage du français l'expression " rire en coin ».

Cl. T. Qu'est-ce que l'humour, pour toi, et de qui tiens-tu ton esprit moqueur?

P. L. Il y a bien des sortes d'humour. La mécanique de l'histoire humoristique est basée sur la surprise. Mais l'humour de l'observation ordinaire est une façon de découvrir ce qui est drôle, inattendu ou surprenant et de le dire d'une manière plaisante. Comme Cyrano, découvrant son pittoresque nez ou Sacha Guitry, dans sa chaise roulante, à qui l'on dit : " Mais ça a l'air de bien aller! » Et qui répond : "Comme sur des roulettes!». L'humour est transgression. C'est pourquoi il est souvent condamné par le politiquement correct et les religions. Une blague sur le Coran, c'est risqué. Il est conseillé de rire de soi ou dans son propre groupe 
culturel plutôt que de se moquer des autres. C'est ce que font si bien les juifs.

J'ai toujours été moqueur. Ça m’a attiré bien des ennuis, dès mon plus jeune âge. C'est un défaut inné!

Cl. T. Reconnais que ces chroniques de L'Express tont valu, parfois, des commentaires pas très flatteurs... T'ont-ils beaucoup dérangé?

P. L. Les réactions hostiles me sont venues de groupes religieux. On m'a même traité d'" Ennemi de l'Humanité », ce qui m’a flatté. J'avais déjà eu ça avec Le Pied de Dieu, lecture irrespectueuse de la Bible. Curieusement, ce sont les religieux fanatiques que je redoutais le plus qui ne m'ont jamais attaqué sérieusement.

Dérangé? Non. J'ai toujours dormi tranquille avec ma conscience.

Cl. T. Un de tes lecteurs t'a accusé de cynisme. Quelle différence fais-tu entre l'humour et le cynisme? et pendant que nous y sommes entre l'humour et lironie?

P. L. L'humour est une figure de style qui peut apparaitre dans n'importe quel genre, gai ou triste. Le cynisme est brutal, anticonformiste et souvent méchant sans être pour cela forcément humoristique. Et l'ironie est une manière de se moquer en ayant l'air de penser comme la personne que l'on veut railler, mais en exagérant de façon telle qu'on est obligé de s'apercevoir de la moquerie. Comme lorsque Swift dit que si l'on mangeait les enfants, on calmerait la faim des pauvres tout en diminuant la surpopulation.

Cl. T. Le grand humoriste Roland Bacri, ancien "Petit Poète " du Canard Enchaîné, t'a fait l'honneur d'une préface où il te nomme son "homologue en coin-coin ». Te reconnais-tu dans l'humour de Bacri?

P. L. Bacri joue essentiellement sur les mots. C'est le roi du calembour. Dans Humour en coin, je ne m'en suis pas privé non plus mais je me suis beaucoup amusé aussi sur l'humour des situations.

Cl. T. Tu joues souvent sur les deux tableaux. Le Papillon à bicyclette est un petit chef-d'ouvre d'humour linguistique, une fête des mots, aussi bien dans les poèmes du début que dans le Bestiaire et les Fables. Comment définirais-tu ce livre de poèmes par rapport à tes chroniques humoristiques? 
P. L. Dans Humour en coin, s'il y a poésie, elle est fortuite. Dans Le Papillon à bicyclette, je crois avoir montré que la poésie peut venir de l'humour, ce qu'André Vanasse a refusé de voir, disant que mon livre était pour les enfants. Oui, mais devenus grands!

Cl. T. Hédi Bouraoui a très bien vu, dans son ouvrage critique, Pierre Léon, Humour et virtuosité, "l'esprit d'enfance " qui caractérise toute ton œuvre. Non?

PAL Oui. Hédi est un bon analyste. Et d'une indulgente gentillesse!

Cl. T. Un autre lecteur, lui aussi auteur et critique littéraire, Ghislain Ripault, écrit sur Humour en coin : "Je tire mon chapeau à tes pieds de nez! " Et il ajoute : "Car tu es une sorte de riant moraliste, loin des bégueuleries, bondieudiantreseries, et autres malapprises à col et cul serrés de tous genres. Et ça enseigne beaucoup sur les bêtises du monde et de certains de ses représentants tous commerce."

As-tu l'impression de moraliser dans tes chroniques?

P. L. S’il y a morale, c'est au lecteur de la trouver. Ça n'est pas difficile quand je parle du prix des indulgences qui ne cesse de monter, comme celui de l'eau bénite. C'est parfois plus compliqué quand il s'agit des escroqueries métaphysiques de l'art moderne. Mais je ne suis certainement pas prêcheur!

Cl. T. Quand on regarde la table des matières de ton livre, on est impressionné par la diversité des thèmes abordés : la logique, les beaux-arts, la séduction, le mariage, le politiquement correct, la politique tout court... jusqu'à la religion. Comment es-tu arrivé à ce très large éventail de sujets?

P. L. Tout simplement en me laissant porter par l'actualité et... l'inspiration du moment!

Cl. T. Ce qui ne me semble pas relever du hasard, c'est la structure de chaque chronique. Il y aurait une étude à faire sur le statut littéraire de la chronique. De la tienne en particulier, et sur son type d'écriture.

P. L. Oui, je pense que la chronique journalistique appartient au genre de la nouvelle. On prend un fait divers, on raconte une histoire et la fin doit 
être une chute inattendue. Je triche parfois en profitant du sujet pour raconter une blague en finale. Mais c'est une chute comme une autre.

Cl. T. En terminant, je tiens à mentionner, pour t'en remercier, le large et chaleureux sourire dont tu m'as gratifié tout au long de cette interview. Nos lecteurs ne l'auront pas vu, évidemment, mais ceux? et celles, bien sûr? qui te connaissent n'auront aucun mal à l'imaginer. Je conclurai en citant Henri Mitterandbonnes blagues, un savoir aussi averti sur toutes choses et toutes gens, de la science des champignons à celle du langage biblique (et jusqu'aux vertus du lait d'autruche ou à celle de faire fortune dans le non-élevage des cochons...) une telle aisance dans le récit et une aussi provocante liberté de pensée et de parole.

P. L. En bref, tu inciterais les estivants à emporter mon bouquin à la plage, comme le fait Liaison?

Cl. T. À la plage comme au cottage! Il est à déguster nimporte où, n’importe quand, ton Humour en coin. Au grand air comme au coin du feu? même entre la poire et le fromage. Merci, Pierre, de tes patientes explications.

\section{Bibliographie}

Humour en coin. Chroniques canadiennes. Toronto : GREF, 2005, $2^{\mathrm{e}}$ édition 2006.

Le Pied de Dieu. Lecture irrespectueuse de la Bible. Toronto : GREF, 2001. Le Papillon à bicyclette. Poâmes, Bestiaire, Fables. Toronto, GREF, 2003. Hédi Bouraoui, Pierre Léon. Humour et virtuosité. Ottawa : Vermillon, 2003. 